\title{
STUDY OF SMALL-SCALE ANISOTROPY OF ULTRA-HIGH-ENERGY COSMIC RAYS OBSERVED IN STEREO BY THE HIGH RESOLUTION FLY'S EYE DETECTOR
}

\author{
R. U. Abbasi, ${ }^{1}$ T. Abu-Zayyad, ${ }^{1}$ J. F. Amann, ${ }^{2}$ G. Archbold, ${ }^{1}$ R. Atkins,,${ }^{1}$ J. A. Bellido, ${ }^{3}$ K. Beloov, ${ }^{1}$ J. W. Belz, ${ }^{4}$ \\ S. BenZvi, ${ }^{5}$ D. R. Bergman, ${ }^{6}$ J. H. Boyer,${ }^{5}$ G. W. Burt, ${ }^{1}$ Z. Cao,,${ }^{1}$ R. W. Clay,${ }^{3}$ B. M. Connolly, ${ }^{5}$ B. R. Dawson, ${ }^{3}$ \\ W. Deng, ${ }^{1}$ Y. Fedorova, ${ }^{1}$ J. Findlay, ${ }^{1}$ C. B. Finley, ${ }^{5}$ W. F. Hanlon, ${ }^{1}$ C. M. Hoffman, ${ }^{2}$ M. H. Holzscheiter,${ }^{2}$ \\ G. A. Hughes, ${ }^{6}$ P. Hüntemeyer, ${ }^{1}$ C. C. H. Jui, ${ }^{1}$ K. Kim,${ }^{1}$ M. A. Kirn, ${ }^{4}$ B. C. Knapp, ${ }^{5}$ E. C. Loh, ${ }^{1}$ M. M. Maestas, ${ }^{1}$ \\ N. Manago, ${ }^{7}$ E. J. Mannel,${ }^{5}$ L. J. Marek, ${ }^{2}$ K. Martens,,${ }^{1}$ J. A. J. Matthews,${ }^{8}$ J. N. Matthews, ${ }^{1}$ A. O’Neill,${ }^{5}$ \\ C. A. Painter, ${ }^{2}$ L. Perera,${ }^{6}$ K. Reil,${ }^{1}$ R. Riehle, ${ }^{1}$ M. D. Roberts,${ }^{8}$ M. Sasaki ${ }^{7}$ S. R. Schnetzer,${ }^{6}$ M. Seman,${ }^{5}$ \\ K. M. Simpson,${ }^{3}$ G. Sinnis, ${ }^{2}$ J. D. Smith,${ }^{1}$ R. Snow,${ }^{1}$ P. Sokolsky, ${ }^{1}$ C. Song,${ }^{5}$ R. W. Springer,${ }^{1}$ B. T. Stokes, ${ }^{1}$ \\ J. R. Thomas, ${ }^{1}$ S. B. Thomas, ${ }^{1}$ G. B. Thomson,${ }^{6}$ D. Tupa, ${ }^{2}$ S. Westerhoff, ${ }^{5}$ L. R. Wiencke, ${ }^{1}$ and A. Zech ${ }^{6}$ \\ (The HiRes Collaboration) \\ Received 2004 April 6; accepted 2004 June 14; published 2004 June 25
}

\begin{abstract}
The High Resolution Fly's Eye (HiRes) experiment is an air fluorescence detector which, operating in stereo mode, has a typical angular resolution of 0.6 and is sensitive to cosmic rays with energies above $10^{18} \mathrm{eV}$. The HiRes cosmic-ray detector is thus an excellent instrument for the study of the arrival directions of ultra-highenergy cosmic rays. We present the results of a search for anisotropies in the distribution of arrival directions on small scales $\left(<5^{\circ}\right)$ and at the highest energies $\left(>10^{19} \mathrm{eV}\right)$. The search is based on data recorded between 1999 December and 2004 January, with a total of 271 events above $10^{19} \mathrm{eV}$. No small-scale anisotropy is found, and the strongest clustering found in the HiRes stereo data is consistent at the 52\% level with the null hypothesis of isotropically distributed arrival directions.
\end{abstract}

Subject headings: acceleration of particles — cosmic rays - large-scale structure of universe

\section{INTRODUCTION}

Identifying the sources of ultra-high-energy cosmic rays remains one of the central challenges in astrophysics. After three decades of systematic searches for the origin of these particles, source identification still remains elusive. Sky maps of cosmicray arrival directions at all energies are generally isotropic, with no obvious source or source region standing out.

A direct way to search for sources of ultra-high-energy cosmic rays is to analyze the distribution of their arrival directions for small-scale clustering. Any significant clustering in arrival directions could be evidence of nearby, compact sources, whereas the lack of clustering is consistent with models in which ultrahigh-energy cosmic-ray sources are distributed at large distances from our Galaxy.

Arrival directions do not necessarily point back to sources, as charged cosmic-ray primaries suffer deflections traveling through Galactic and intergalactic magnetic fields. The strength and orientation of these fields is not well established, so the size and direction of the deflection are difficult to ascertain. However, since the Larmor radius increases with energy, the

\footnotetext{
${ }^{1}$ University of Utah, Department of Physics and High Energy Astrophysics Institute, Salt Lake City, UT 84112.

${ }^{2}$ Los Alamos National Laboratory, P.O. Box 1663, Los Alamos, NM 87545.

${ }^{3}$ University of Adelaide, Department of Physics, Adelaide, SA 5005, Australia.

${ }^{4}$ University of Montana, Department of Physics and Astronomy, Missoula, MT 59812.

${ }^{5}$ Columbia University, Department of Physics and Nevis Laboratories, New York, NY 10027; finley@phys.columbia.edu, westerhoff@nevis.columbia.edu.

${ }^{6}$ Rutgers, The State University of New Jersey, Department of Physics and Astronomy, 136 Frelinghuysen Road, Piscataway, NJ 08854-8019.

${ }^{7}$ University of Tokyo, Institute for Cosmic Ray Research, 515 Kashiwano-Ha, Kashiwa City, Chiba 277-8582, Japan.

${ }^{8}$ University of New Mexico, Department of Physics and Astronomy, Albuquerque, NM 87131.
}

possibility of observing small-scale anisotropy associated with cosmic rays pointing back to their origins is expected to grow.

Indeed, small-scale clustering of cosmic-ray arrival directions at the highest energies has been previously claimed. The AGASA (Akeno Giant Air Shower Array) experiment reported possible clustering in their sample of events with energies above $4 \times 10^{19} \mathrm{eV}$ (Hayashida et al. 1996). The analysis has been updated several times (Takeda et al. 1999, 2001; Teshima et al. 2003), most recently reporting six clusters (five doublets and one triplet) in a sample of 59 events, where a cluster is defined as a set of events with angular separation less than 2.5 . The chance probability of this signal was reported to be less than $10^{-4}$ (Teshima et al. 2003).

Given the potential importance of this result for our understanding of the origin of cosmic rays, it is crucial to test the claim that clustering is a feature of cosmic-ray arrival directions with independent experimental data. Since 1999, the High Resolution Fly's Eye (HiRes) air fluorescence experiment has been operating in stereo mode, collecting data of unprecedented quality on the arrival direction, energy, and composition of ultra-high-energy cosmic rays. In this Letter, we report results of a search for small-scale anisotropy in the arrival directions of ultra-high-energy cosmic rays observed by the HiRes stereo detector between 1999 December and 2004 January.

\section{THE HIRES DETECTOR}

HiRes is an air fluorescence experiment with two sites (HiRes 1 and 2) at the US Army Dugway Proving Ground in the Utah desert $\left(112^{\circ}\right.$ west longitude, $40^{\circ}$ north latitude, with a vertical atmospheric depth of $860 \mathrm{~g} \mathrm{~cm}^{-2}$ ). The two sites are separated by a distance of $12.6 \mathrm{~km}$.

Each of the two HiRes "eyes" comprises several telescope units monitoring different parts of the night sky. With 22 (42) telescopes with 256 photomultiplier tubes each at the first (sec- 


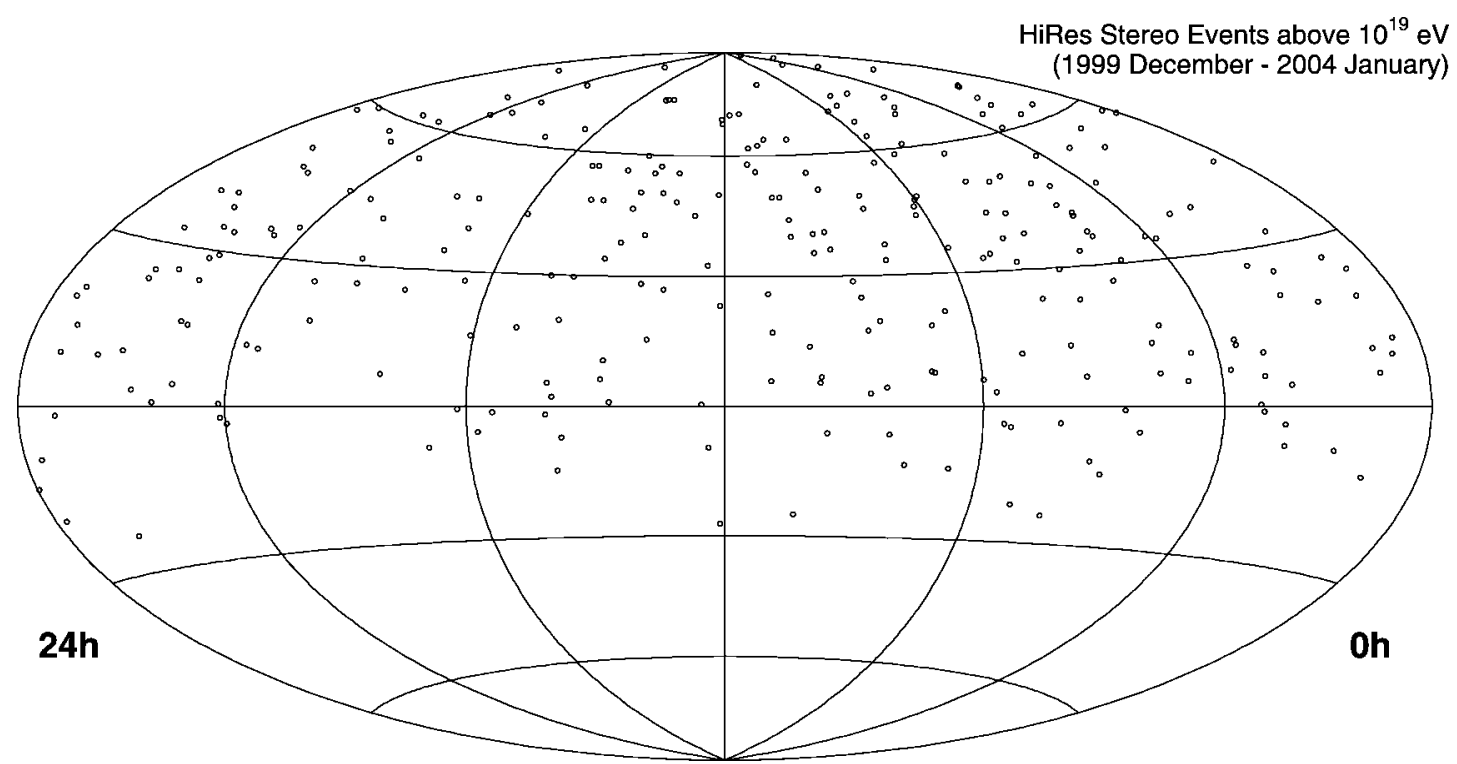

FIG. 1.-Sky map (in equatorial coordinates) of the 271 HiRes stereo events above $10^{19} \mathrm{eV}$ examined in this study. The typical error radius of 0.6 is used for all events.

ond) site, the full detector covers about $360^{\circ}\left(336^{\circ}\right)$ in azimuth and $3^{\circ}-16.5\left(3^{\circ}-30^{\circ}\right)$ in elevation above the horizon. Each telescope consists of a mirror with an area of about $5 \mathrm{~m}^{2}$ for light collection and a cluster of photomultiplier tubes in the focal plane.

A cosmic-ray primary interacting in the upper atmosphere induces an extensive air shower that the detectors observe as it develops through the atmosphere. The photomultiplier tubes triggered by the shower define an arc on the sky, and, together with the position of the detector, the arc determines the socalled shower-detector plane. When an air shower is observed in stereo, the shower trajectory is in principle simply the intersection of the two planes. This method can be further improved by also taking advantage of the timing information of the tubes, and in our analysis the shower geometry is determined by a global $\chi^{2}$ minimization using both the timing and pointing information of all tubes. From measurements of laser tracks and stars in the field of view of the cameras, we estimate
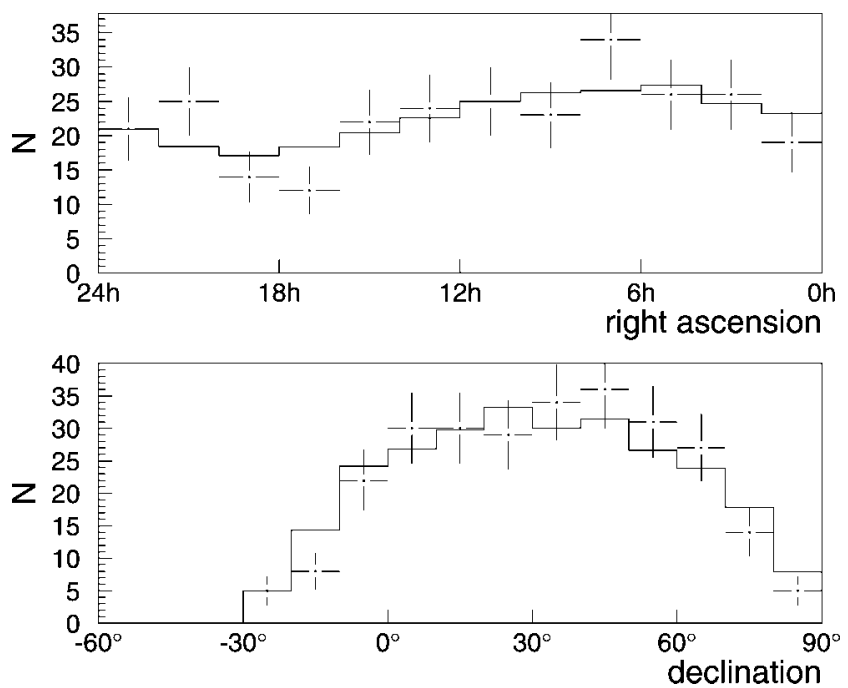

FIG. 2.-Right ascension and declination of events above $10^{19} \mathrm{eV}$ observed from 1999 December through 2004 January. The points with error bars represent the data, and the solid line represents the Monte Carlo events. For right ascension, $\chi^{2} / \mathrm{dof}=0.77$; for declination, $\chi^{2} / \mathrm{dof}=0.73$. that the systematic error in the arrival direction determination is no larger than 0.2 and is mainly caused by uncertainties in the survey of mirror pointing directions. Various aspects of the HiRes detector and the reconstruction procedures are described in Boyer et al. (2002), Sadowski et al. (2002), and Matthews et al. (2003).

While a ground array detector can operate year-round, night and day, air fluorescence detectors can only be operated on dark, moonless nights with good atmospheric conditions. This limits the duty cycle to about $10 \%$. However, several years of observation yield a data set with a relatively smooth distribution in sidereal time, modulated by an overall seasonal variation in exposure.

For the present analysis, we subject the HiRes stereo event sample to the following quality cuts. We require a minimum track length of $3^{\circ}$ in each detector, an estimated angular uncertainty in both the azimuth and zenith angles of less than $2^{\circ}$, and a zenith angle of less than $70^{\circ}$. We additionally require an estimated energy uncertainty of less than $20 \%$ and a $\chi^{2}$ per degree of freedom (dof) of less than 5 for both the energy and

TABLE 1

Results for Simulated Clusters

\begin{tabular}{|c|c|c|c|c|}
\hline \multirow[b]{2}{*}{$m$} & \multicolumn{2}{|c|}{$\sigma_{R}=0.6$} & \multicolumn{2}{|c|}{$\sigma_{R}=1.8$} \\
\hline & Median $P_{\mathrm{ch}}$ & $90 \% P_{\mathrm{ch}}$ & Median $P_{\mathrm{ch}}$ & $90 \% P_{\mathrm{cl}}$ \\
\hline \multicolumn{5}{|c|}{$N_{H}=27$} \\
\hline $2 \ldots \ldots$ & 0.018 & 0.090 & 0.13 & 0.48 \\
\hline $3 \ldots \ldots$ & $2.5 \times 10^{-3}$ & 0.013 & 0.050 & 0.25 \\
\hline $4 \ldots \ldots$ & $3.1 \times 10^{-4}$ & $1.5 \times 10^{-3}$ & 0.016 & 0.11 \\
\hline \multicolumn{5}{|c|}{$N_{H}=47$} \\
\hline $3 \ldots \ldots$ & 0.011 & 0.067 & 0.12 & 0.47 \\
\hline $4 \ldots \ldots$ & $1.9 \times 10^{-3}$ & 0.012 & 0.059 & 0.32 \\
\hline $5 \ldots \ldots$ & $3.3 \times 10^{-4}$ & $2.2 \times 10^{-3}$ & 0.029 & 0.18 \\
\hline \multicolumn{5}{|c|}{$N_{H}=89$} \\
\hline $4 \ldots \ldots$ & 0.016 & 0.11 & 0.16 & 0.59 \\
\hline $6 \ldots \ldots$ & $1.0 \times 10^{-3}$ & 0.012 & 0.071 & 0.38 \\
\hline $8 \ldots \ldots$ & $1.1 \times 10^{-4}$ & $7.3 \times 10^{-4}$ & 0.025 & 0.20 \\
\hline
\end{tabular}

Note. $-N_{H}=27,47$, and 89 events corresponds to simulated clustering above energy thresholds 40, 28, and $20 \mathrm{EeV}$, respectively. 


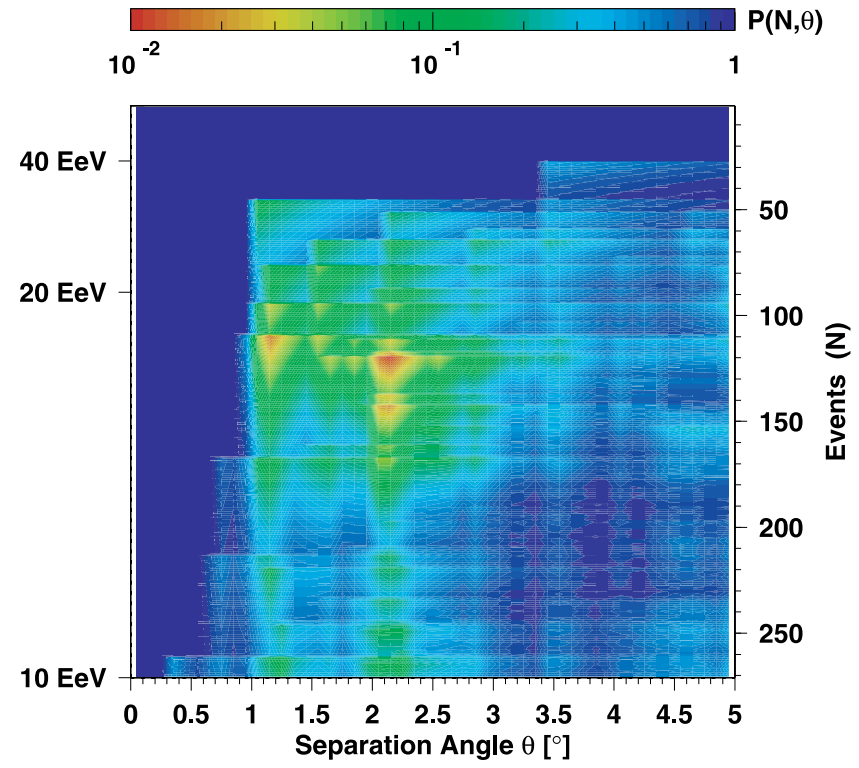

FIG. 3.-Autocorrelation scan of the HiRes data set above $10^{19} \mathrm{eV}$. $P(N, \theta)$ is the probability of obtaining the same or greater number of pairs as is actually observed in the data using a maximum separation angle $\theta$ and searching among the $N$ highest energy events. These probabilities do not include the statistical penalty due to scanning.

the geometry fit. Weather conditions that reduce the quality of the data are cut implicitly in the above sample, rather than by explicit weather cuts. A total of 271 events above $10^{19} \mathrm{eV}$ pass the selection criteria. A sky map in equatorial coordinates of the arrival directions of these events is shown in Figure 1.

The angular resolution of HiRes is determined using simulated showers. We use a full detector simulation of proton showers generated with CORSIKA 6 (Heck et al. 1998) using QGSJET for the first interaction. Applying the same cuts to the simulation data that are applied to the real data, $68 \%$ of all showers generated at $10^{19} \mathrm{eV}$ are reconstructed within less than 0.57 of the true shower direction. The angular resolution depends weakly on energy, with the $68 \%$ error radius growing to 0.61 and 0.69 for showers generated at $4 \times 10^{19} \mathrm{eV}$ and $10^{20} \mathrm{eV}$, respectively, because at higher energy, showers are on average farther away. The angular resolution is essentially constant in the zenith and azimuth angles of the arrival direction, varying by less than $0{ }^{\circ} 1$.

Using the same simulation described above, we generate an isotropic distribution of showers with a differential spectral index $\alpha=-3.0$ in energy, and we use the resulting distribution of reconstructed Monte Carlo events to determine the detector acceptance in zenith and azimuth. We then randomly match the local coordinates of these events with times during which the detector was operating in order to generate an exposure map in equatorial coordinates. Figure 2 shows the distributions of the data and Monte Carlo events in right ascension and declination.

\section{METHOD}

We search for small-scale clustering by performing an autocorrelation scan in energy and angular separation. Essentially, we consider the set of $N$ events above energy $E$, count the number of pairs $n_{p}$ separated by less than $\theta$, and evaluate the probability $P(N, \theta)$ of finding this number or more pairs, given $N$ and $\theta$. We repeat this for a range of values for $E$ and $\theta$, and use the smallest probability $P_{\min }$ found in the scan to identify the strongest clustering signal. We estimate the statistical significance $P_{\text {ch }}$ of this signal by performing identical scans over simulated sets of isotropically distributed data, counting the fraction of simulated sets that yield the same or smaller value for $P_{\min }$.

The virtue of this approach is that by letting the energy threshold vary, we let the scan itself determine the optimal balance between the better statistics of the low-energy data set and the (presumably) smaller angular deflections at high energies. Furthermore, we can simultaneously look for clustering both at the angular scale identified by AGASA and at smaller scales that take advantage of the HiRes angular resolution. The statistical penalty for performing multiple searches is accounted for in the final evaluation of the significance $P_{\mathrm{ch}}$. We note that, just as in the usual two-point correlation function, higher order multiplets are counted by the individual number of pairs that they contain.

To determine the probabilities $P(N, \theta)$, we generate a large number of simulated data sets (typically $10^{7}$ ) corresponding to an isotropic distribution of cosmic rays. Specifically, we generate an event with a random arrival direction in equatorial coordinates, and we accept that event into the simulated data set with a probability proportional to the HiRes exposure in that region of the sky. We then construct a table of values $P_{\mathrm{MC}}$, where $P_{\mathrm{MC}}(N, \theta, n)$ is the fraction of data sets in which the first $N$ events contain exactly $n$ pairs separated by less than $\theta$. Then the probability $P(N, \theta)$ for observing $n_{p}$ or more pairs at $(N, \theta)$ is simply

$$
P(N, \theta)=\sum_{n=n_{p}}^{\infty} P_{\mathrm{MC}}(N, \theta, n)=1-\sum_{n=0}^{n_{p}-1} P_{\mathrm{MC}}(N, \theta, n)
$$

For some combination $N_{c}$ and $\theta_{c}, P$ has a minimum: $P_{\min }=$ $P\left(N_{c}, \theta_{c}\right)$. We identify this as the strongest potential clustering signal. To determine the statistical significance, we perform the same scan over $n_{\mathrm{MC}}$ Monte Carlo data sets, finding the minimum probability $P_{\min }^{i}=P^{i}\left(N_{c}^{i}, \theta_{c}^{i}\right)$ for each trial and counting the number of trials $n_{\mathrm{MC}}^{*}$ for which $P_{\min }^{i} \leq P_{\min }$. The significance is finally identified as

$$
P_{\mathrm{ch}}=\frac{n_{\mathrm{MC}}^{*}}{n_{\mathrm{MC}}}
$$

that is, the chance probability of observing the value $P_{\min }$ or less in an isotropic distribution.

The scan is performed over the total set of $N=271$ events and over angular separations $\theta$ from $0^{\circ}$ to $5^{\circ}$ in increments $\Delta \theta$ of 0.1 . Rather than use an arbitrary fixed increment $\Delta E$ of energy, we increase the energy threshold one event at a time $(\Delta N=1)$. These search parameters were chosen a priori. While the results inevitably depend on the exact choices, the dependence is relatively small (see Finley \& Westerhoff 2004 for details and examples).

To demonstrate the effectiveness of this method and the sensitivity of the HiRes detector, we apply this technique to simulated data with clusters. First, we generate a set of 271 events with the HiRes exposure for isotropic arrival directions. We then insert $m$ pairs of events among the $N_{H}$ highest energy events in the set to simulate clustering above a specific energy threshold.

To create a pair, we pick a point in the sky for the source location and generate two events with arrival directions deviating from the source location according to a Gaussian distri- 
bution described below. These artificial cluster positions are chosen at random, but their distribution is forced to reflect the overall exposure of the HiRes detector, so that regions with higher exposure are more likely to contain a cluster. The pair of events is then added to the original isotropic data set, replacing two of the original events in the set. This is repeated until $m$ pairs have been inserted. The set may contain more than $m$ pairs due to chance.

For simplicity, we use a circular Gaussian distribution for the smearing of arrival directions around the source location. The width of the distribution $\sigma_{R}$ can be set equal to the angular resolution of the detector, or it can be set to a larger value to simulate additional smearing by magnetic fields. [Note that for the Gaussian distribution $P(\theta)=\left(\theta / \sigma^{2}\right) e^{-\theta^{2} / 2 \sigma^{2}}$, the value $\theta=$ $1.515 \sigma$ encloses $68 \%$ of the distribution. We therefore define $\left.\sigma_{R}=1.515 \sigma.\right]$

Table 1 shows the results of these simulations using the detector resolution $\left(\sigma_{R}=0.6\right)$ as well as 3 times the detector resolution $\left(\sigma_{R}=1.8\right)$ to simulate additional smearing by magnetic fields. For each choice of $N_{H}, m$, and $\sigma_{R}$, we generate $10^{4}$ data sets and scan them with the procedure described above to find a distribution of values for the significance $P_{\mathrm{ch}}$. The median and 90th percentile values of this distribution are indicated in Table 1.

The table shows, for example, that for a clustering signal on the $\sigma_{R}=0.6$ scale, even three pairs among the 47 highest energy events would typically result in $P_{\mathrm{ch}}=1.1 \%$. The table also shows that three such pairs would result in $P_{\mathrm{ch}}<6.7 \%$ for $90 \%$ of the simulated sets. Thus, an actual value of $P_{\mathrm{ch}}>$ $6.7 \%$ could be used to exclude the possibility that sources contributed three such pairs at more than the $90 \%$ confidence level. These results demonstrate the sensitivity to clustering on small angular scales.

\section{RESULTS AND DISCUSSION}

We perform the scan on the HiRes stereo sample of 271 events above $10^{19} \mathrm{eV}$. Because we start well below the $4 \times$ $10^{19} \mathrm{eV}$ energy associated with the AGASA clustering signal, our search should safely encompass the energy region of interest even in the presence of a systematic energy shift of $30 \%$ between the two experiments, as suggested by De Marco et al. (2003). Starting at this energy does not appreciably dilute the significance of a clustering signal if one is found at higher energy, since the scan involves repeated searching with successively higher energy thresholds. An additional motivation for starting at $10^{19} \mathrm{eV}$ is the fact that the HiRes angular resolution $(0.6)$ is much sharper at this energy than AGASA's (2.8; Takeda et al. 1999).

The results of the scan are shown in Figure 3. The strongest clustering signal $\left(P_{\min }=1.9 \%\right)$ is observed using the energy threshold $E_{c}=1.69 \times 10^{19} \mathrm{eV}$ where we observe $n_{p}=10$ pairs separated by less than $\theta_{c}=2.2$ within a set of $N_{c}=$ 120 events. The statistical significance of this result corresponds to $P_{\text {ch }}=52 \%$. The HiRes stereo data above $10^{19} \mathrm{eV}$ are therefore consistent with the null hypothesis of isotropic arrival directions.

Comparison with the AGASA clustering result is not straightforward. The HiRes stereo event sample above $4 \times 10^{19} \mathrm{eV}$ is still smaller than AGASA's, although how much smaller depends critically on the level of agreement in absolute energy scale for the two experiments. The possibility of a systematic energy shift of $30 \%$ would imply that above the rescaled energy threshold, $(0.7) \times 4 \times 10^{19} \mathrm{eV}=2.8 \times 10^{19} \mathrm{eV}$, HiRes has seen 47, rather than 27 , events. More importantly, there is the question of how many pairs an independent data set might be expected to contain, given the lack of an obvious source model and the widely varying estimates of the strength of the AGASA clustering. Without assuming a model and source strength, there is no natural way to translate the AGASA observation of five doublets and one triplet separated by less than 2.5 into a meaningful prediction for HiRes.

However, what can be tested using a statistically independent data set is the claim that significant small-scale clustering is a general feature of ultra-high-energy cosmic-ray arrival directions. The HiRes stereo data set does not support such a claim. We observe no statistically significant evidence of clustering on any angular scale up to $5^{\circ}$ at any energy threshold above $10^{19} \mathrm{eV}$.

Comparing the observed value of $P_{\mathrm{ch}}$ with the values obtained from simulations in $\S 3$ (shown in Table 1), we note that if the current HiRes data above $4 \times 10^{19} \mathrm{eV}$ contained two or more pairs of events contributed by compact sources at the angular resolution limit of the detector, then the typical value of $P_{\mathrm{ch}}$ would be 0.018 or less, and more than $90 \%$ of the time the value of $P_{\mathrm{ch}}$ would be much smaller than the observed value of 0.52 .

Results of searches for correlations with known astrophysical source classes will be published in a separate paper.

The HiRes project is supported by the National Science Foundation under contracts NSF-PHY-9321949, NSF-PHY9322298, NSF-PHY-9974537, NSF-PHY-0098826, and NSFPHY-0245428, by the Department of Energy grant FG0392ER40732, and by the Australian Research Council. The cooperation of Colonels E. Fisher and G. Harter and of the US Army and Dugway Proving Ground staff is appreciated. We thank the authors of CORSIKA for providing us with the simulation code.

\section{REFERENCES}

Boyer, J., et al. 2002, Nucl. Instrum. Methods Phys. Res. A, 482, 457 De Marco, D., Blasi, P., \& Olinto, A. V. 2003, Astropart. Phys., 20, 53 Finley, C. B., \& Westerhoff, S. 2004, Astropart. Phys., 21, 359

Hayashida, N., et al. 1996, Phys. Rev. Lett., 77, 1000

Heck, D., et al. 1998, CORSIKA: A Monte Carlo Code to Simulate Extensive Air Showers (Wissenschaftliche Berichte FZKA 6019; Karlsruhe: Forschungszentrum Karlsruhe)
Matthews, J. N., et al. 2003, Proc. 28th Int. Cosmic Ray Conf. (Tsukuba), 350 Sadowski, P. A., et al. 2002, Astropart. Phys., 18, 237

Takeda, M., et al. 1999, ApJ, 522, 225

- 2001, Proc. 27th Int. Cosmic Ray Conf. (Hamburg), 341

Teshima, M., et al. 2003, Proc. 28th Int. Cosmic Ray Conf. (Tsukuba), 341 